\title{
Quantifying Uncertainties for Prostate Image-Guided Radiotherapy: A 3D Organ Reconstruction and Registration Method
}

\author{
Ximo Gual-Arnau ${ }^{*}, 1$, Rubén Masó ${ }^{1}$, Françoise Lliso ${ }^{2}$ and Juan López-Tarjuelo ${ }^{3}$ \\ ${ }^{I}$ Department of Mathematics, Universitat Jaume I, 12071-Castelló, Spain \\ ${ }^{2}$ Servicio de Oncología Radioterápica, Hospital Universitario La Fe, Valencia, Spain \\ ${ }^{3}$ Servicio de Radiofísica y Protección Radiológica, Instituto Oncológico, C.H. Provincial de Castellón, Castellón, Spain
}

\begin{abstract}
The purpose of this paper is to present a method for volumetric reconstruction, registration and margin assignation applicable to both conventional CT scans and on board CT imaging. This method does not depend on the shape of the organs, the bony anatomy or the use of markers, and we apply it to prostate and bladder. 3D reconstructions are performed by means of spline surfaces and the 3D reconstructed surfaces are registered to a planning surface, using a multidimensional alignment from the Euclidean distance transform and the Levenberg-Marquardt optimization algorithm. Once the reconstructed surfaces are registered, we define a mean surface and obtain the corresponding variances from this mean surface. The method works properly and demonstrates that once translations are insulated by registration, residual uncertainties can be handled with the margin assigned for delineation variation and organ deformation.
\end{abstract}

Keywords: Geometrical uncertainties, image-guided radiotherapy, interobserver variability, organ motion, registration, shape variation, stereology, volumetric reconstruction.

\section{INTRODUCTION}

The success of radiotherapy relies on the accurate delivery of the prescribed dose to the tumor, while sparing the surrounding organs at risk to a maximum extent. Geometrical uncertainties in radiotherapy exist due to variability and uncertainties in organ contouring, often caused by the modality of imaging used, uncertainties introduced by treatment planning system, and setup uncertainties and organ translations, rotations and deformations during the treatment. Although different correction procedures have been introduced to minimize the effects of treatment variation (electronic portal imaging devices (EPID), the implanted markers, repeated computed tomography scans (CT), and on board CT's, etc.) like, a margin around the clinical target volume (CTV) will always remain necessary because of intrafraction organ motion, residual setup uncertainties, and target delineation inaccuracies.

Great effort has been made to develop interfraction organ registration and to assess the better margin to CTV. Present trends aim to use of the organ registration to a prior image of that organ, to correct changes and misalignments, instead of the use of bony anatomy. In addition, volumetric registration, through a $3 \mathrm{D}$ reconstruction, must be desirable in order to consider translations and rotations, and the evaluation of appropriate margin in a volumetric fashion. Therefore, the method we present accomplishes those requirements and can be applied to both on board cone beam CT's (CBCT) and CT scans outside the treatment room.

*Address correspondence to this author at the Department of Mathematics, Universitat Jaume I, 12071-Castelló, Spain; E-mail: gual@mat.uji.es
On the other hand, the problem of assessing organ variations has been extensively studied in the literature (see, for instance, $[1,2]$ ), but few methodologies exist for 3D analysis of organ deformation $[3,4]$.

This paper is a continuation of [5], where we presented a study to assess the uncertainties that arise in locating the boundaries of anatomical structures. As a conclusion of [5], we considered to use three dimensional reconstructed images from the CT scans, to obtain the treatment margins.

Our procedure, for the quantification of organ motion (including setup errors and internal organ motions) and shape variation of organs, does not depend on the shape of organs, the bony anatomy or the use of markers, and as an example we apply it to prostate and bladder. The surface reconstruction from the CT scans is based on splines surfaces and the registration between the repeated reconstructed surfaces was done by using a multidimensional alignment from the Euclidean distance transform and the LevenbergMarquardt optimization algorithm [6]. However, since the conventional approach to correct organ motion is patient repositioning by a simple translation, we apply the above mentioned algorithms for translations alignment of the surfaces instead of rigid transformations. Then, by using 3D mean surfaces, distances perpendicular to the mean surfaces and the best ellipsoid that fits the standard deviations of the perpendicular distances, we compared what we will call 'local errors', which include shape variation and rotations (due to patient positioning and internal organ motions), with the translation of the organs. The intraobserver variation is also included in the 'local errors' and we do not treat it separately. 
We also apply this method to quantify the interobserver variability and we compare this interobserver variability with the 'local errors' and translations.

Moreover, we report a stereological study for prostate and bladder volume, which includes an error approximation of this estimation depending on the number of CT slices.

\section{MATERIAL AND METHODS}

\subsection{Material}

The medical data consist of CT parallel sections of the pelvic region of a patient with prostate carcinoma. To determine the 'local errors' (organ rotations, shape variations and intraobserver variability) compared with the translations of the organs, from seven different CT studies of the same patient, and from the manually delineated contours of the organs in all sections of each CT study, we obtained seven reconstructed three-dimensional surfaces (prostate and bladder) corresponding to each of the 7 CT studies.

The CT sections were acquired during 7 different days and the prostate and bladder were delineated on each scan by the same observer. The slice thickness and spacing was 10 $\mathrm{mm}$.

To study the interobserver variability, the contours of the organs in all sections of one of the seven CT studies were manually delineated by 4 independent observers.

Therefore, for the interobserver variability study, from the CT images of one CT study we have 4 surfaces (contours) for each organ (prostate and bladder) corresponding to the four observers, and for the 'local errors' and translations determination, from the $\mathrm{CT}$ images of each of the 7 CT studies we have $73 \mathrm{D}$ surfaces corresponding to the same observer.

In order to obtain standard deviation functions and margins, we applied the mathematical and statistical methodology, detailed in section 2.2, to these sets of 7 and 4 surfaces.

\subsection{Methods}

\section{Matching of 3D Surfaces}

From the CT scans of each CT study a 3D surface reconstruction for prostate and bladder is obtained, based on splines surfaces. That is, from each CT scan we choose 40 equidistant points of the organ delineated boundary, then from all the selected points in all CT scans the reconstructed surface is obtained by using B-Splines. This reconstruction method works properly for general shapes, not only for star or nearly spherical shapes.

One of the CT scans is considered as the planning scan, and therefore, the corresponding $3 \mathrm{D}$ reconstructed surface is considered as the planning surface. If another CT scan is considered to be the planning surface, no relevant differences in the registration results are obtained.

Then, each of the other 3D reconstructed surfaces are registered to the planning surface using a multidimensional alignment from the Euclidean distance transform and the Levenberg-Marquardt optimization algorithm [6]. However, we apply these algorithms for translations alignment of the surfaces instead of for rigid transformations. This is because we want to compare 'local errors', which include shape varia- tion and rotations (due to patient positioning and internal organ motions), with the translation of the organs. The registration method we use does not depend either on bony anatomy or the use of markers and gives us a measure of the translations between the CT scans.

\section{Mean Surfaces and Standard Deviations}

To define a mean surface of all the registered 3D surfaces, we consider a finite number of perpendicular vectors to the planning surface. Now, we consider the distances along the lines defined by these perpendicular vectors, from the planning surface to each of the registered surfaces. The points which give the mean distance in each perpendicular line are used to obtain the mean surface by B-Splines.

In addition, we have a standard deviation function $\mathrm{SD}_{u}$, which depends on the perpendicular vector $u$ and provides us a local variation between the registered $3 \mathrm{D}$ surfaces with respect to the mean surface. From these local variations, we can obtain treatment margins in all directions, however, we only take the anterior-posterior (AP), left-right (LR), and superior-inferior (SI) directions, because only these directions are commonly considered in clinical practice, and in order to compare our results with other studies existing in the literature relative to variability in the radiotherapy process, we will take the best-fitting ellipsoid (see, for instance, [7, 8] to the standard deviation function $\mathrm{SD}_{u}$. Then, from the axis of the ellipsoid and the corresponding transformation (from the maximum values) to the coordinate axis, we also obtain the AP, LR, and SI variations.

\section{Volume Estimation}

Since the prostate volume has an impact on the treatment regime for the patients [9], we will estimate the prostate and bladder volumes by $\mathrm{CT}$, using the Cavalieri method. The Cavalieri volume estimation formula is given by the product between the CT section thickness and the total sectional area of the consecutive sections of the target organ (prostate or bladder). The coefficient of error (CE) of this method has been extensively studied in the literature [10]. We will calculate the approximated $\mathrm{CE}$ using equation (15) of [5], and we will compare this $\mathrm{CE}$ with the coefficient of variation among organ volumes of the different CT studies.

\section{RESULTS}

\subsection{Local Errors and Translations Determination}

The first of the seven CT studies of a patient with prostate cancer was considered as the planning scan. The reconstructed prostate and bladder surfaces, corresponding to the other six CT studies, were registered to the planning surface using a translation alignment. In Table 1, we have the modulus of the six translation vectors (all measures are in $\mathrm{cm}$ ) and the mean modulus of the vectors.

A mean surface of seven matched prostate and bladder surfaces was obtained. Fig. (1) shows the planning prostate and the mean prostate. The standard deviation function of the registered prostate surfaces with respect to the mean prostate surface, for different perpendicular vectors, is plotted in Fig. (2a). The best-fitting ellipsoid to the standard deviation function is obtained (see Fig. 2b), and Table 2 shows the leftright (LR), anterior-posterior (AP) and superior-inferior (SI) variations obtained from the axes of the ellipsoid and the 
transformations to the coordinate axes, using the maximum of the ellipsoid axes.

(a)

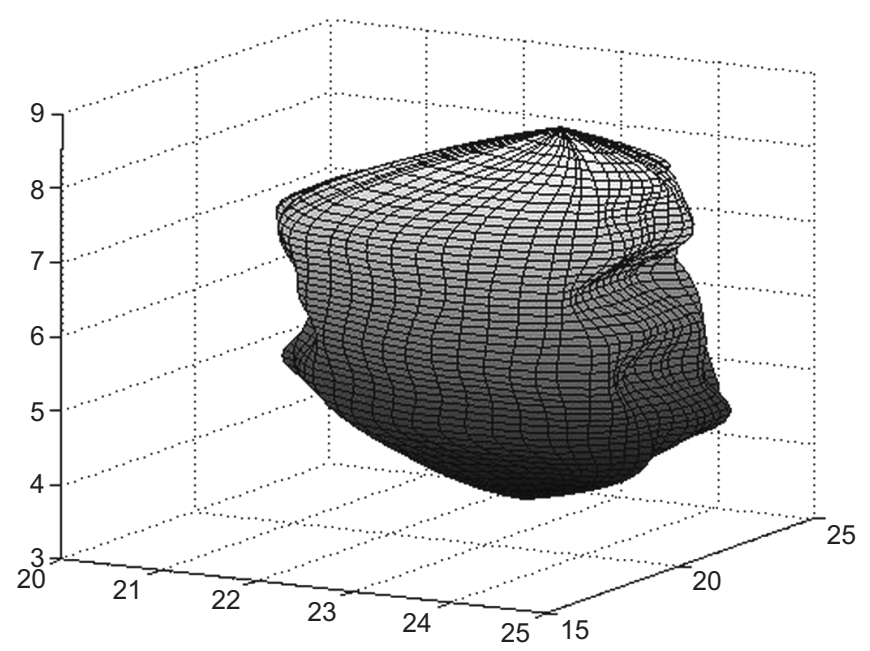

(b)

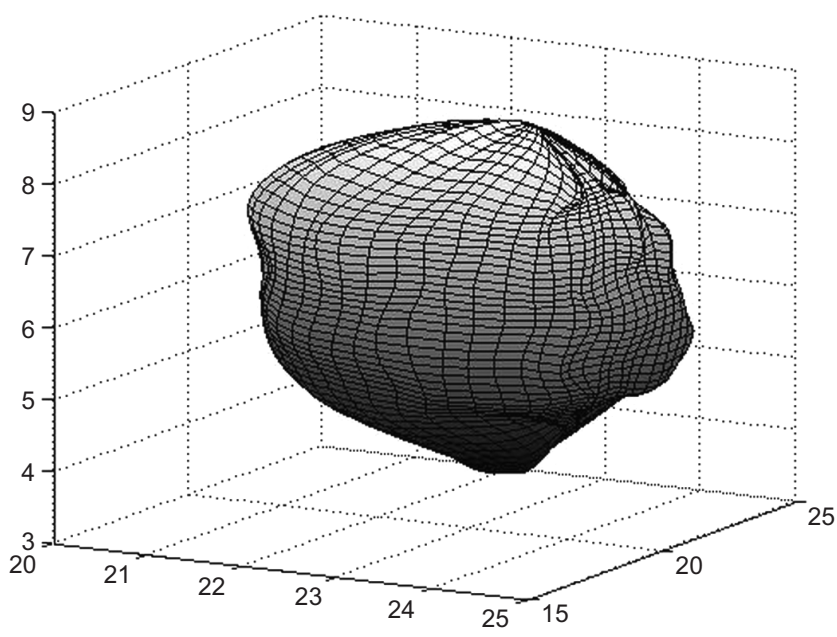

Fig. (1). 3D planning prostate and mean prostate.

In order to examine the prostate and bladder volumes assessed by the Cavalieri method, Table 3 shows the mean coefficient of error of the Cavalieri estimations of the prostate and bladder volumes, and the coefficient of variation with respect to the mean volume of prostate and volume.

\subsection{Interobserver Variability}

For the interobserver variability study, we have four curves (contours) for each organ (prostate and bladder) in each CT image of one of the CT studies, corresponding to four independent observers. Therefore, we have four reconstructed surfaces and a mean surface (the process of matching is not needed in this case). Now, as in the preceding section, Table 2 shows the LR, AP and SI variations and Table 3 shows the mean coefficient of error of the volume estimation of organs and the coefficient of variation with respect to the mean volume obtained from the four observers estimation.

\section{DISCUSSION}

External beam radiotherapy represents a treatment option for the patients with prostate cancer, where computed tomo- graphy (CT) is by far the most widely used form of imaging. In fact, one of the major subprocesses in external therapy is treatment planning, which includes CT image acquisition and delineation of tumor volume and organs at risk on the CT images. In order to know the relative magnitude of different geometrical uncertainties, we develop a method to quantify organ motions, shape variations, setup errors and interobserver variability, valid for different kinds of shapes and which does not depend on bony anatomy or the use of markers.

(a)

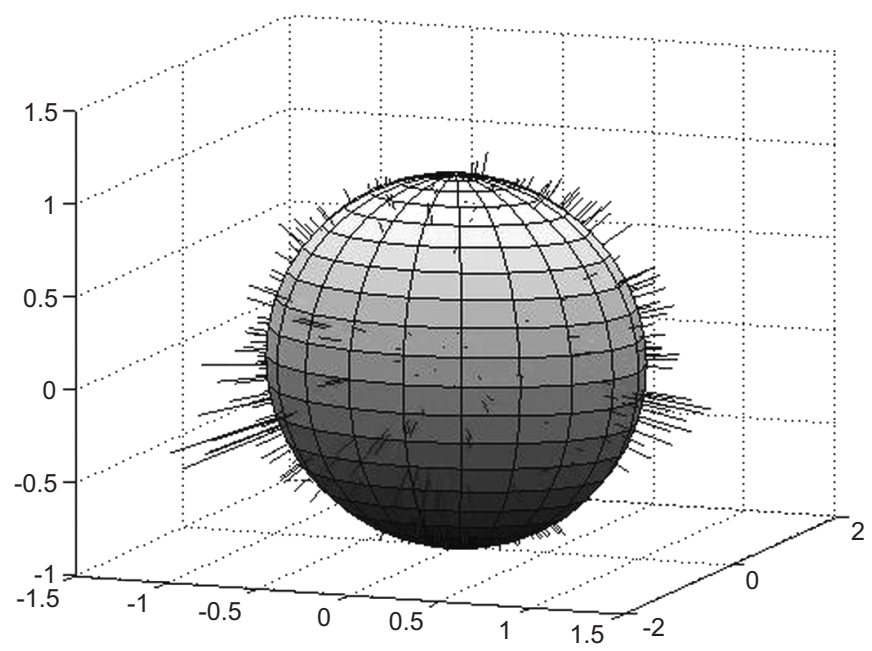

(b)

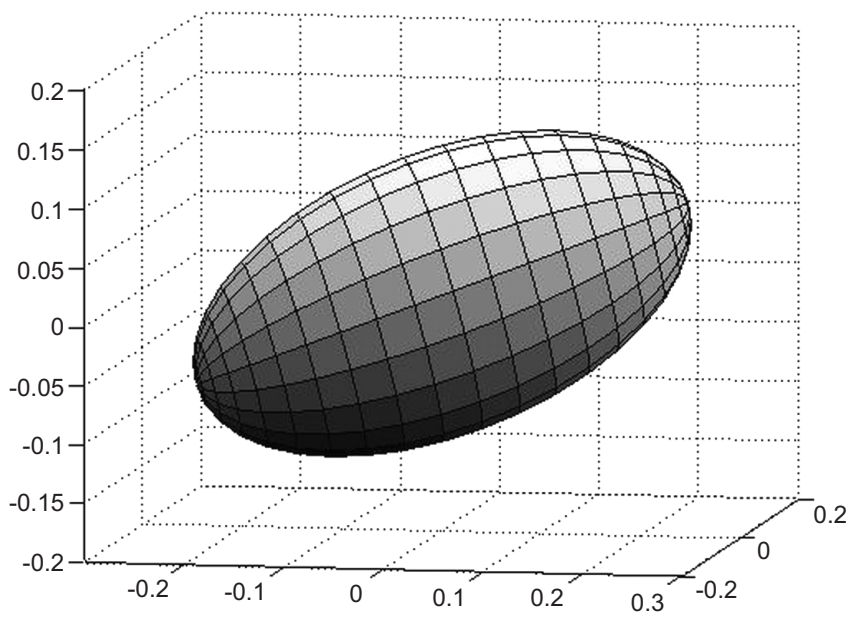

Fig. (2). Standard deviation function from the mean surface and the best-fitting ellipsoid to the standard deviation function.

Most of the studies present in the literature separately consider the setup errors and the organ motion, that is, first, each of the scans is registered to the planning scan (using a bone match, external markers,...), and next a 3D target volume (tumor volume, organs at risk,...) match is performed using translations and rotations. With our method, the organ motion study includes setup errors and internal organ motion and we analyze the magnitude of translations of the volumes as compared with rotations and shape variations. However, our results are consistent with findings from other studies, see for instance [4] and references therein. 
Table 1. Magnitude of the Translation Vectors from Each Reconstructed Surface to the Planning Surface

\begin{tabular}{|c|c|c|c|c|c|c|c|}
\hline Prostate & CT n. 2 & CT n. 3 & CT n. 4 & CT n. 5 & CT n. 6 & CT n. 7 & Mean \\
\hline \hline Modulus (in cm) & 1,3156 & 1,8836 & 1,2881 & 1,8976 & 1,3028 & 0,81004 & 1,41629 \\
\hline Bladder & CT n. 2 & CT n. 3 & CT n. 4 & CT n. 5 & CT n. 6 & CT n. 7 & Mean \\
\hline \hline Modulus (in cm) & 1,5413 & 2,1292 & 1,2454 & 1,914 & 1,0298 & 0,87348 & 1,45553 \\
\hline
\end{tabular}

Table 2. Variation (in $\mathrm{cm}$ ) in the Three Main Directions, After Translations Alignment in the Local Errors Study, Obtained from the Best-Fitting Ellipsoid to the Standard Deviation Function

\begin{tabular}{|c|c|c|c|}
\hline & LR & AP & SI \\
\hline \hline Prostate (local errors) & 0,219 & 0,142 & 0,1 \\
\hline Bladder (local errors) & 0,241 & 0,267 & 0,156 \\
\hline Prostate (interobserver variability) & 0,188 & 0,121 & 0,112 \\
\hline Bladder (interobserver variability) & 0,266 & 0,332 & 0,945 \\
\hline
\end{tabular}

Although the aim of this paper is to present a new method for volumetric reconstruction, registration and margin assignation, from the results summarized in Tables $\mathbf{1}$ and 2 it is shown that local errors and interobserver variability are small, if we compare them with the organs translation. The SI variation for the bladder interobserver variability is near to one $(0,945)$ because in this study we only considered slice thickness of $1 \mathrm{~cm}$, therefore, the SI variations in the interobserver variability may be highly influenced by the contours delineated by the observers in each CT images. It may happens that one observer delineate bladder contour in one CT image where another observer does not observe bladder.

Therefore, if this conclusion is ratified with more exhaustive and detailed clinical studies, we think that in order to reduce the effects of the geometrical uncertainties in external radiotherapy it is important, in first order, to correct the translation (displacement) of organs (which includes setup misalignment) and prostate and bladder rotation and deformation, and the interobserver variability, can be considered as a second-order effect.

Therefore, although there is no definite system or procedure that can be regarded as standard for treatment verification in external radiotherapy [12], when it will be possible to correct prostate translations with correction protocols as verification images (EPID, CBCT, ...), the appropriate margins in all directions to correct shape variation, rotation and interobserver variabilities will reduce considerably.

Table 3. Coefficient of Error of the Stereological Volume Estimation Using the Cavalieri Method

\begin{tabular}{|c|c|c|}
\hline & Mean $\mathbf{C E}$ & CV \\
\hline \hline Prostate (local errors) & 0.028 & 0.117 \\
\hline Bladder (local errors) & 0.019 & 0.179 \\
\hline Prostate (interobserver variability) & 0.028 & 0.048 \\
\hline Bladder (interobserver variability) & 0.026 & 0.042 \\
\hline
\end{tabular}

There exist several methods to estimate prostate volume (see [9] and references therein). One of the advantages of the Cavalieri method is that it provides an unbiased estimation of volume and there exist formulas to predict the coefficient of error of this estimation [11]. In Table 3, we have the mean coefficient of error of this estimator when applied to prostate and bladder. If we compare this value with the coefficient of variation of volumes with respect to the mean volume, we observe a great difference between these values in the local errors study, but the difference is not large in the interobserver variability. The main reason for this is because all the reconstructed structures correspond to the same CT session in the interobserver variability study, however, in the local error study the seven structures correspond to seven different CT sessions and there exist differences in the applied protocols (between the different sessions) to control the rectum and bladder filling, etc.

\section{CONCLUSION}

We have developed a method to quantify the organ motion (including setup errors and internal organ motions), shape variation of organs, and interobserver variability, in the radiotherapy process. This method has been applied to prostate and bladder and it is based on a translation alignment of 3D surfaces, to correct organs displacement, using multidimensional alignment from the Euclidean distance transform and the Levenberg-Marquardt optimization algorithm, and the use of 3D mean surfaces, distances perpendicular to the mean surfaces and the best ellipsoid that fits the standard deviations of the perpendicular distances, to obtain margins in the main directions.

This method may also be applied for rigid motions and not only for translations, however, due to the shape of prostate and bladder (more or less round) the rotations of these organs are not always easy to analyze.

The purpose of this paper is to present a model to use in the treatment plan; a discussion about the feasibility of the presented approach in real clinical scenario should be done in clinical studies to ensure the advantages of the model.

\section{ACKNOWLEDGEMENT}

This work was supported by a DGES Grant MTM200508689-C02-02 and the Bancaixa Foundation Grant P11B2008-23. The medical images were provided by the 'Sección de Oncología Radioterápica. Hospital la Fe de Valencia'.

\section{REFERENCES}

[1] Melian E, Mageras GS, Fucks Z. Variation in prostate position quantification and implications for three-dimensional conformal treatment planning. Int J Radiat Oncol Biol Phys 1997; 38: 73-81. 
[2] Van-Herk M, Bruce A, Kroes APG. Quantification of organ motion during conformal radiotherapy of the prostate by three dimensional (3d) image registration. Int J Radiat Oncol Biol Phys 1995; 33: 1311-20.

[3] Remeijer P, Rasch C, Lebesque JV, Van Herk M. A general methodology for three-dimensional analysis of variation in target volume delineation. Med Phys 1999; 26(6): 931-40.

[4] Deurloo KEI, Steenbakkers R, Zijp LJ, et al. Quantification of shape variation of prostate and seminal vesicles during external beam radiotherapy. Int J Radiat Oncol Biol Phys 2005; 61(1): 22838.

[5] Gual-Arnau X, Ibañez-Gual MV, LLiso F, Roldán S. Organ contouring for prostate cancer: Interobserver and internal organ motion variability. Comput Med Imag Grap 2005; 29: 639-47.

[6] Kozinska D, Tretiak OJ, Nissanov J, Ozturk C. Multidimensional alignment using the euclidean distance transform. Graph models image proc 1997; 59: 373-87.
[7] Banegas F, Jaeger M, Michelucci D, Roelens M. The ellipsoidal skeleton in medical applications. In Proceedings of the sixth ACM symposium on solid modeling and applications, ACM Press 2001; 193: 30-8.

[8] Jang-Condell H, Hernquist L. First structure formation: A simulation of small-scale structure at high redshift. Astrophys J 2001; 548: 68-78.

[9] Kalkner KM, Kubicek G, Nilsson J, Lundell M, Levitt S, Nilsson S. Prostate volume determination: Differential volume measurements comparing ct and trus. Radiother Oncol 2006; 81: 179-83.

[10] Vedel Jensen EB. Local Stereology. World Scientific Pub Co Inc; 1998.

[11] Cruz-Orive LM. Precision of cavalieri sections and slices with local errors. J Microsc 1999; 193: 182-98.

[12] Boehmer D, Maingon P, Poortmans P, et al. Guidelines for primary radiotherapy of patients with prostate cancer. Radiother Oncol 2006; 79: 259-69.

(C) Gual-Arnau et al.; Licensee Bentham Open.

This is an open access article licensed under the terms of the Creative Commons Attribution Non-Commercial License (http://creativecommons.org/licenses/by$\mathrm{nc} / 3.0 /$ ) which permits unrestricted, non-commercial use, distribution and reproduction in any medium, provided the work is properly cited. 